\title{
Fever after intraventricular neuroendoscopic procedures in children
}

\author{
S. L. de Kunder ${ }^{1}$ - M. P. ter Laak - Poort ${ }^{1}$ - J. Nicolai ${ }^{2}$ - J. S. H. Vles ${ }^{2}$ • E. M. J. Cornips ${ }^{1}$
}

Received: 22 March 2016/Accepted: 6 April 2016/Published online: 14 April 2016

(C) The Author(s) 2016. This article is published with open access at Springerlink.com

\begin{abstract}
Purpose The purpose of this paper was to study the incidence and clinical significance of fever after intraventricular neuroendoscopic procedures in children.

Methods We retrospectively assessed all children subjected to an intraventricular neuroendoscopic procedure between 2004 and 2015. Body temperature 6 days postoperatively, symptoms and signs, and eventual cerebrospinal fluid analysis were evaluated. Fever was defined as temperature above $38^{\circ} \mathrm{C}$. Results Fifty-five children (mean age 4.8 years) had 67 procedures. Forty-three children (47 procedures, $70 \%$ ) developed fever, mostly the day of surgery $(n=17 ; 25 \%)$ or the next day $(n=33 ; 49 \%)$. All children who were clinically ill ( $n=9$, including 7 with fever) suffered serious illness, as opposed to none of the children with fever without being clinically ill $(n=36)$. Fever was unrelated to gender, indication for, and type of procedure and did not influence ETV success rate at 3 months. Children under 1 year less frequently developed fever $(p=0.032)$.

Conclusions Fever frequently develops after intraventricular neuroendoscopic procedures in children and follows a rather predictable course, peaking the day of surgery and/or the next day, and rapidly subsiding thereafter. Fever is not a cardinal symptom except when combined with other symptoms in children who are clinically ill (which most of them are not). Close observation avoiding invasive diagnostic tests may suffice for those who are not clinically ill, while extra attention should be
\end{abstract}

E. M. J. Cornips

E.cornips@mumc.nl

1 Department of Neurosurgery, Maastricht University Medical Center, Maastricht, The Netherlands

2 Department of Child Neurology, Maastricht University Medical Center, Maastricht, The Netherlands paid to those whose temperature rises after day 2 especially when clinically ill, as they likely suffer serious illness. We recommend to closely observe children after any intraventricular neuroendoscopic procedure for at least 5 days.

Keywords Children $\cdot$ Complications $\cdot$ Endoscopic third ventriculostomy $\cdot$ Neuroendoscopy $\cdot$ Postoperative fever
Abbreviations
C Celsius
CSF Cerebrospinal fluid
ETV Endoscopic third ventriculostomy

\section{Introduction}

Neuroendoscopic procedures have proven to be a reliable and safe alternative to open procedures [1-3]. Fever after intraventricular neuroendoscopic procedures is frequently observed, but not extensively discussed in the literature. Postoperative fever always causes a dilemma for the attending physician who has to decide whether (mostly invasive) diagnostic tests are warranted to determine the cause of the fever, or whether watchful waiting is an option. Of note, young children with chronic medical problems who are subjected to invasive procedures are at greater risk for complications such as dehydration and occult bacteremia as compared to adults [4]. In addition, they are more prone to an imbalance between heat production and heat dissipation, and therefore more vulnerable. For these reasons, the question if and when postoperative fever points to a serious illness is even more relevant in (young) children than in adults. According to a recent paper by Kinoshita et al. [5], the incidence of fever 
after intraventricular neuroendoscopic procedures in children under 10 years of age may be as high as $84.4 \%$. The purpose of this paper was to study the incidence and clinical significance of fever after intraventricular neuroendoscopic procedures in children, hoping a better understanding of this phenomenon will help the attending physicians to avoid unnecessary and especially invasive diagnostic procedures.

\section{Material and methods}

This retrospective research is not subject to the Medical Research Involving Human Subjects Act in the Netherlands (WMO in Dutch). All children (less than 18 years old at time of surgery) subjected to an intraventricular neuroendoscopic procedure in our institute between January 2004 and February 2015 were retrospectively analyzed. Children with fever or signs of infection prior to surgery were excluded. All data were obtained from medical records, including such surgical details as the exact kind of procedure, duration of surgery, irrigation fluid used, and eventual complications. Body temperature was measured three times a day (morning, afternoon, and evening) using a tympanic membrane thermometer for at least 6 days postoperatively (the minimum stay according to local protocol). Children with fever (defined as a temperature above $38{ }^{\circ} \mathrm{C}$ ) were assigned to two categories: those with a temperature in between 38 and $39{ }^{\circ} \mathrm{C}$ (mild fever) and those with a temperature above $39{ }^{\circ} \mathrm{C}$ (high fever). Clinical symptoms and signs were collected from all children with fever in order to find out whether or not they were clinically ill. ETV success rate was assessed 3 months postoperatively.

\section{Surgical technique}

All procedures were performed under general anesthesia using a peel-away sheath and stylet to puncture the ventricle. The scope used was either a rigid fiberscope (Clarus scope, Medtronic, Minneapolis, USA) or a rodlens scope (Minop scope, B. Braun - Aesculap, Melsungen, Germany). Ringer's lactate solution at body temperature was invariably used as irrigation fluid. Perioperative prophylactic antibiotics were administered to all children (flucloxacillin/rifampicin in those $<6$ months old and cefazolin in those $>6$ months old).

\section{Statistical analysis}

All data were processed and analyzed with the Statistical Package for the Social Sciences (IBM SPSS Statistics, version 22). Data were tested for normal distribution using a Kolmogorov-Smirnov test and a Shapiro-Wilk test, while a
Student's $t$ test was used to compare means, and a chi-square test to compare percentages. Results were expressed as means and standard deviation or percentages. A $p$ value $<0.05$ was considered statistically significant.

\section{Results}

\section{Patient characteristics and surgical details}

Table 1 summarizes patient characteristics and surgical details. A total of 62 children had a total of 76 procedures, of which 9 procedures were excluded because of insufficient data due to the retrospective character of the study. The remaining 67 procedures were performed in predominantly male subjects ( $66 \%$ male vs. $34 \%$ female) with an average age of 4.8 years $(0-15$; sd 5.4$)$. Indications for surgery were aqueductal stenosis, 4th ventricular outflow problem including Blake's pouch cyst, Dandy-Walker malformation, arachnoid cyst, intra-axial tumor, or other (Figs. 1 and 2).

Table 2 summarizes ETV success rate at 3 months, divided into two age groups (those under 1 year, and those above 1 year) as it has been well established that ETV success rate is substantially lower in children under 1 year [2]. All children had ETV or re-ETV, in most cases $(n=54 ; 81 \%)$ as an isolated procedure. In few cases, ETV was combined with cyst puncture $(n=6 ; 9 \%)$ or tumor biopsy $(n=7 ; 10 \%)$. Average duration of surgery was 58 minutes (16-150; sd 27). In this series, we encountered one major complication, a severe intraventricular hemorrhage in a child who subsequently developed permanent hypothalamic dysfunction. There was no mortality associated with the neuroendoscopic procedures.

Table 1 Patient characteristics and surgical details

\begin{tabular}{ll}
\hline Number of children & 55 \\
\hline $\begin{array}{l}\text { Number of procedures } \\
\text { Gender }\end{array}$ & 67 \\
Male & $44(66 \%)$ \\
Female & $23(34 \%)$ \\
Age (years) & $4.8(0-15$, sd 5.4$)$ \\
Indication for surgery & \\
Aqueductal stenosis & $32(48 \%)$ \\
4th ventricular outflow problem/Blake's pouch cyst & $12(18 \%)$ \\
Dandy Walker malformation & $3(5 \%)$ \\
Arachnoid cyst & $7(10 \%)$ \\
Tumor & $12(18 \%)$ \\
Other & $1(1 \%)$ \\
Kind of procedure & $54(81 \%)$ \\
ETV & $7(10 \%)$ \\
ETV and tumor biopsy & $6(9 \%)$ \\
ETV and cyst puncture & $58(16-150$, sd 27$)$ \\
Duration of surgery (min) & \\
\hline
\end{tabular}

${ }^{\mathrm{a}}$ Including re-ETV 

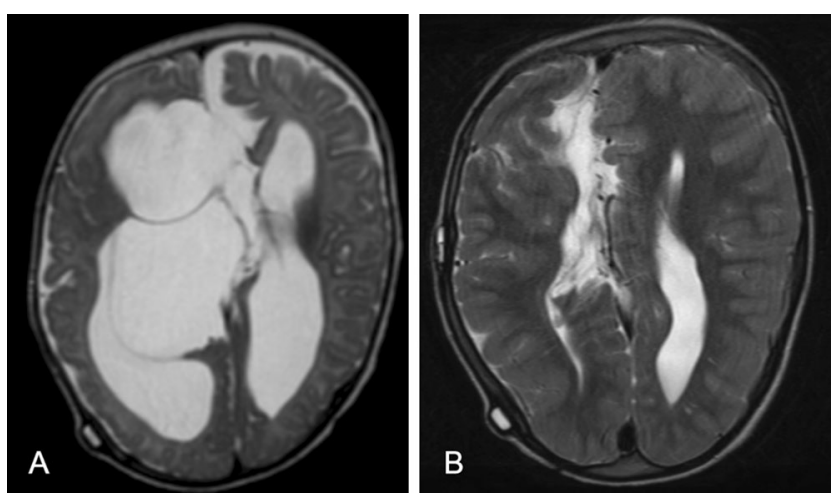

Fig. 1 Pre- (a) and postoperative (b) axial T2-weighted MR images of a child with posthemorrhagic cystic ventricular dilatation and no longer functional ventriculoperitoneal shunt

\section{Postoperative fever}

Details are summarized in Table 3. During the specified follow-up period ( 6 days postoperatively), 43 children (47 (70\%) of all neuroendoscopic procedures) developed a temperature above $38.0{ }^{\circ} \mathrm{C}$, and 15 of them $(22 \%$ of all neuroendoscopic procedures) developed a temperature above $39.0^{\circ} \mathrm{C}$. Most children developed fever quite early, more specifically the day of surgery (day 0 ) or the next day (day 1 ). In the former group, 17 children (25\% of all neuroendoscopic procedures) had a temperature above $38.0^{\circ} \mathrm{C}$, including 2 (3\%) above $39.0{ }^{\circ} \mathrm{C}$, while in the latter group, 33 children
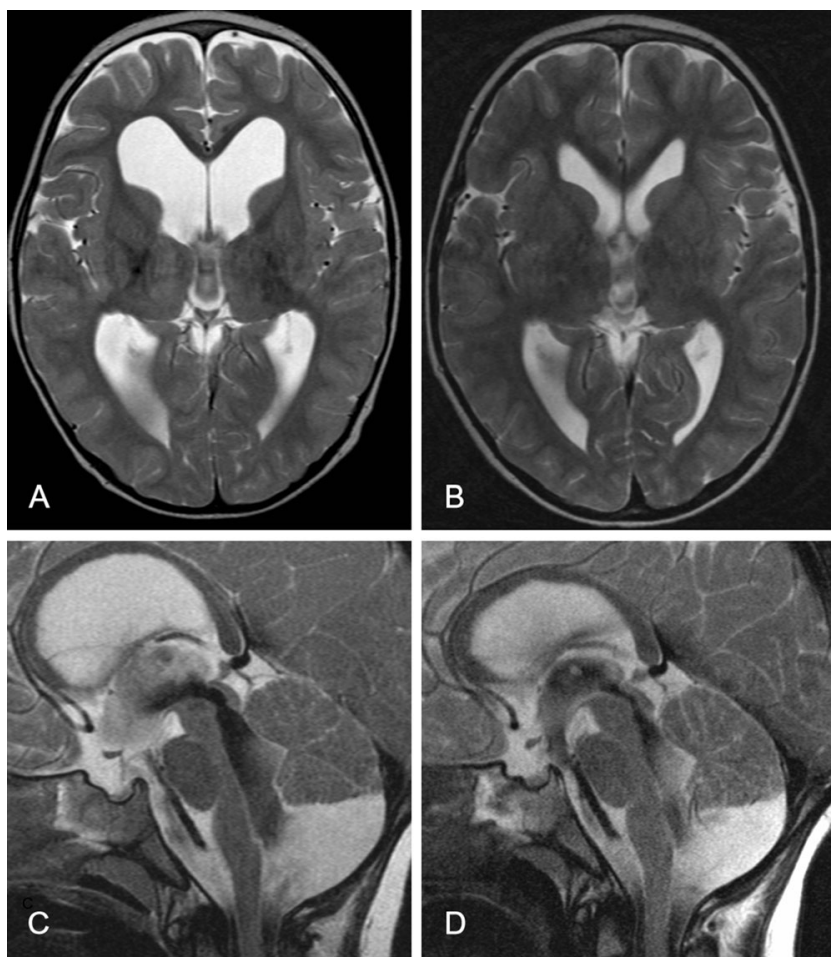

Fig. 2 Preoperative axial (a) and sagittal (c) T2-weighted MR images of a child with Blake's pouch cyst. Axial (b) and sagittal (d) T2-weighted MR images after ETV

Table 2 ETV success rate at 3 months

\begin{tabular}{llll}
\hline & Under 1 year & Above 1 year & Overall \\
\hline ETV success rate (3 months) & $52 \%$ & $71 \%$ & $61 \%$
\end{tabular}

(49\% of all neuroendoscopic procedures) had a temperature above $38.0{ }^{\circ} \mathrm{C}$, including $11\left(16 \%\right.$ ) above $39.0^{\circ} \mathrm{C}$ (Fig. 3). Merely 9 children (13\% of 67 procedures) were clinically ill according to the attending physician's assessment, most of them having a fever $(n=7 ; 78 \%)$, including 3 with a temperature between 38.0 and $39.0{ }^{\circ} \mathrm{C}$, and 4 with a temperature above $39.0{ }^{\circ} \mathrm{C}$ (Table 4$)$.

\section{Clinically ill children}

Nine children were observed to be clinically ill, whereas the remaining ones were fine. More specifically, 9 out of 67 procedures $(13 \%)$ were followed by an episode of clinical illness. Four children demonstrated a lowered level of consciousness within 48 hours of the neuroendoscopic procedure. All of them were diagnosed with an acute hydrocephalus (early ETV failure) requiring re-ETV, external ventricular drainage, or internal shunting. Four other children demonstrated nuchal rigidity. One of them previously treated for a thalamic anaplastic astrocytoma was suffering dramatic tumor progression and died within 1 week after the procedure. The other three were suspect for postoperative meningitis and therefore subjected to a lumbar puncture. In two of them, cerebrospinal fluid (CSF) analysis was consistent with bacterial meningitis (low glucose and elevated protein levels); however, in merely one of them, the gram stain was positive and actual bacteria (Pneumococcus) were cultured, while the other one may have had aseptic meningitis, or CSF cultures may have been sterilized due to the perioperative prophylactic antibiotics. Based on their clinical presentation and abnormal CSF analysis, however, both children had broad-spectrum antibiotics for possible bacterial meningitis for a period of 12 days. The third child with reassuring CSF analysis (normal glucose and protein levels) was subjected to an MRI scan because of persisting illness, demonstrating an intraventricular hemorrhage as the likely

Table 3 Postoperative fever ( 67 procedures in 55 children)

\begin{tabular}{lll}
\hline & $>38.0{ }^{\circ} \mathrm{C}^{*}$ & $>39.0{ }^{\circ} \mathrm{C}$ \\
\hline Day 0 & $17(25 \%)$ & $2(3 \%)$ \\
Day 1 & $33(49 \%)$ & $11(16 \%)$ \\
Day 2 & $17(25 \%)$ & $2(3 \%)$ \\
Day 3 & $10(15 \%)$ & $2(3 \%)$ \\
Day 4 & $7(10 \%)$ & 0 \\
Day 5 & $2(3 \%)$ & $2(3 \%)$ \\
Day 6 & $4(6 \%)$ & 0 \\
\hline
\end{tabular}

*Including those with fever $>39.0^{\circ} \mathrm{C}$ 
Fig. 3 Incidence of postoperative fever

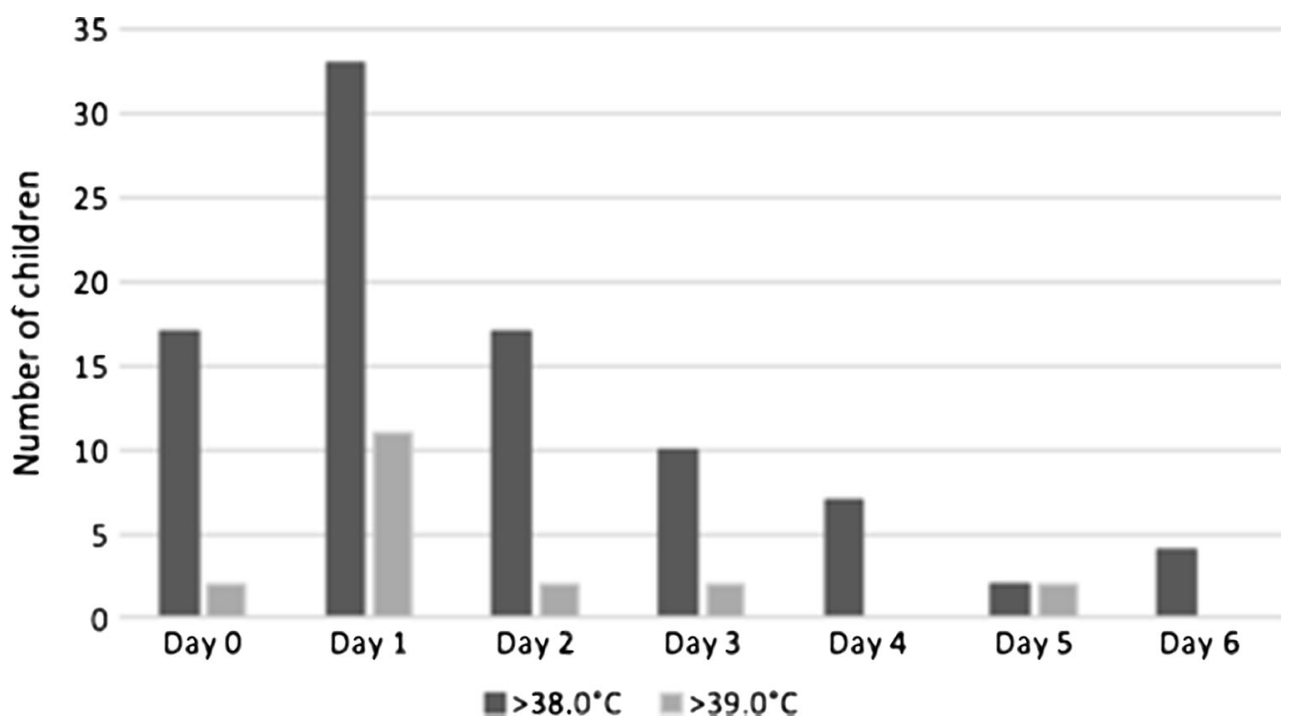

cause for its symptoms. Another child had symptoms consistent with gastroenteritis and was treated accordingly (without antibiotics). Interestingly, while many children (70\%) had fever on day 0 and/or day 1 after the neuroendoscopic procedure, those who went on to develop an infection (either a meningitis or a gastroenteritis) demonstrated a secondary rise in temperature after day 2. Importantly, we did not observe meningitis or any other infectious disease in any of the children with fever (even above $39^{\circ} \mathrm{C}$ ) who were not clinically ill.

\section{Variables predictive for postoperative fever}

There was no significant relation between postoperative fever and gender, indication for, and type of intraventricular neuroendoscopic procedure. Moreover, postoperative fever did not seem to influence ETV success rate at 3 months. However, we observed a relation with age, as children under 1 year $(n=27 ; 67 \%)$ were less likely to develop postoperative fever $(p=0.032)$. Moreover, we observed a trend towards significance for duration of surgery, as children with a procedure under 58 minutes (the mean duration of surgery in this series) were more likely to develop postoperative fever $(p=0.056)$ (Table 5). Temperature rising above $38.0{ }^{\circ} \mathrm{C} 3$ to 6 days postoperatively more frequently occurred in clinically ill children ( 5 out of 9 children, $56 \%$ ) as compared to non-clinically ill children ( 9 out of 58 children, $16 \%$ ) and was usually indicative for a serious illness.

\section{Discussion}

Fever (defined as a temperature above $38.0{ }^{\circ} \mathrm{C}$ ) after intraventricular neuroendoscopic procedures in both pediatric and adult patients is likely underreported. In several papers on intraventricular neuroendoscopy, transient fever is merely mentioned as frequently occurring postoperatively, but not discussed in any detail [6-9]. Kinoshita et al. [5] recently reported an incidence of $65.1 \%$ in a mixed pediatric and adult patient series, including an incidence of $84.4 \%$ in children under 10 years, whereas we observed an incidence of $70 \%$ (47 out of 67 procedures)

Table 4 Clinically ill children

\begin{tabular}{|c|c|c|c|c|}
\hline & No fever & $>38.0{ }^{\circ} \mathrm{C}$ & $>39.0^{\circ} \mathrm{C}$ & Underlying condition \\
\hline Child no. 1 & & • & $\cdot$ & Meningitis \\
\hline Child no. 2 & & - & - & Meningitis \\
\hline Child no. 3 & & - & & Acute hydrocephalus ${ }^{\mathrm{a}}$ \\
\hline Child no. 4 & & - & & Acute hydrocephalus ${ }^{\mathrm{a}}$ \\
\hline Child no. 5 & & - & & Tumor progression \\
\hline Child no. 6 & & - & - & Gastroenteritis \\
\hline Child no. 7 & & - & - & Intraventricular hemorrhage \\
\hline Child no. 8 & • & & & Acute hydrocephalus ${ }^{\mathrm{a}}$ \\
\hline Child no. 9 & - & & & Acute hydrocephalus ${ }^{\mathrm{a}}$ \\
\hline
\end{tabular}

${ }^{\text {a }}$ Indicating early ETV failure 
Table 5 Variables predictive for postoperative fever

\begin{tabular}{|c|c|c|c|}
\hline & No fever $(n=20)$ & Fever $(n=47)$ & $p$ value \\
\hline \multicolumn{4}{|l|}{ Gender } \\
\hline Male & 14 & 30 & 0.633 \\
\hline Female & 6 & 17 & \\
\hline Age (years) & & & 0.032 \\
\hline Under 1 year & 12 & 15 & \\
\hline Above 1 year & 8 & 32 & \\
\hline Indication for surgery & & & 0.941 \\
\hline Aqueductal stenosis & 11 & 21 & \\
\hline 4th ventricular outflow problem/Blake's pouch cyst & 1 & 11 & \\
\hline Dandy Walker malformation & 2 & 1 & \\
\hline Arachnoid cyst & 2 & 5 & \\
\hline Tumor & 4 & 8 & \\
\hline Other & 0 & 1 & \\
\hline Kind of procedure & & & 0.694 \\
\hline $\mathrm{ETV}^{\mathrm{a}}$ & 17 & 37 & \\
\hline ETV and tumor biopsy & 2 & 5 & \\
\hline ETV and cyst puncture & 1 & 5 & \\
\hline Duration of surgery (min) & & & 0.056 \\
\hline Under $58^{\prime}$ & 8 & 31 & \\
\hline Above $58^{\prime}$ & 12 & 16 & \\
\hline ETV success rate ( 3 months) & 10 & 31 & 0.156 \\
\hline
\end{tabular}

${ }^{\mathrm{a}}$ Including re-ETV in an exclusively pediatric patient series $(n=62)$. Although postoperative fever (even above $39.0{ }^{\circ} \mathrm{C}$ ) does not seem to influence ETV success rate at 3 months, it is important to better understand the phenomenon. Hence, unnecessary and especially invasive diagnostic procedures may be avoided, and parents, pediatricians, and other caregivers may be reassured, even when the exact pathophysiological mechanism has not yet been elucidated [10].

Body temperature is the result of a delicate balance between heat production and heat dissipation [11], coordinated by a complex system involving temperature regulating centers situated in the hypothalamus. A large number of heat- and cold-sensitive neurons in the preoptic nucleus of the anterior hypothalamus function as temperature sensors. Whenever the preoptic nucleus is heated, the body reacts with profuse sweating and excess body heat production is inhibited. At the posterior hypothalamus, peripheral thermal input from the skin is processed and combined with signals from the preoptic nucleus of the anterior hypothalamus to maintain a balanced body temperature $[12,13]$. In this regard, Chernov et al. [10] postulated that fever after intraventricular neuroendoscopic procedures may be the result of irritation of the hypothalamus due to mechanical stimulation (while perforating the third ventricular floor), small hemorrhages, and/or air in the ventricular system. On a cellular level, cytokines play an important role in the development of fever. Several animal studies have shown that the direct injection of certain cytokines, especially interleukin-1 (IL-1) and prostaglandin $\mathrm{E}_{2}$, in the hypothalamus results in an immediate or delayed temperature rise [13, 14]. Of note, children are different from adults as their bloodbrain barrier may still be immature and their developing brain is not the strictly controlled, stable environment an adult brain is [15]. We hypothesize an intraventricular neuroendoscopic procedure may easily cause a disturbance in this vulnerable environment contributing to the development of fever.

Fever was most frequently observed the day of surgery $(n=17 ; 25 \%)$ or the next day $(n=33 ; 49 \%)$ in our patient series. In this early postoperative period, four patients demonstrated a lowered level of consciousness, and all of them were diagnosed with an acute hydrocephalus (early ETV failure) requiring another neurosurgical intervention. As merely two of them (50\%) had a fever, which is less than the overall incidence in our patient series $(n=47$; $70 \%$ ), early postoperative fever is not indicative for early ETV failure. Likewise, postoperative fever (even above $39.0{ }^{\circ} \mathrm{C}$ ) does not seem to influence ETV success rate at 3 months (61\% overall ETV success rate, $66 \%$ in those with and $50 \%$ in those without postoperative fever, the latter group including $60 \%$ children under 1 year) (Table 5). Most of these 
children with fever on day 0 and/or day 1 had a rapid subsidence within the next few days and an otherwise uneventful postoperative course (Fig. 3).

Fever was less frequently observed more than 2 days postoperatively ( $n=14 ; 21 \%$ of 67 procedures). Five children (36\%) were clinically ill, and all of them were soon diagnosed with a serious illness, including meningitis (one bacterial, one possibly aseptic), gastroenteritis, tumor progression, or intraventricular hemorrhage (one child each). Nine children (64\%) were not clinically ill, and all of them had an otherwise uneventful postoperative course.

Comparing our series to the series recently reported by Kinoshita et al. [5], we observe some similarities as well as some differences. Of note, these authors present a series of both adults and children with a mean age of 25.7 (sd 24.0) and a median age of 14.0 years (range 0.2 86.3), whereas we present an exclusively pediatric series. Dividing their series in two age groups, the incidence of postoperative fever in patients under 10 years was $84.4 \%$, and in those above it was $52.8 \%$. In our series, with a mean age of 4.8 years $(0-15$; sd 5.4$)$, the overall incidence of postoperative fever was $70 \%(n=47)$. More specifically, the incidence was $56 \%$ in children under 1 year and $80 \%$ in children above 1 year (Table 5). Of note, the latter incidence is almost identical to the one reported by Kinoshita et al. [5]. Other differences between both series involve the duration of surgery and the irrigation fluid used. Whereas our average duration of surgery was 58 minutes (median 50 minutes), their average duration of surgery was 105.5 minutes (median 102 minutes). Interestingly, while in our series a shorter procedure seems to correlate with an increased incidence of postoperative fever (30/38 under $58^{\prime}$ versus $16 / 28$ above $58^{\prime}$, $p=0.056$, Table 5), the substantial difference in the duration of surgery between our series and the Kinoshita series (almost twice as long) does not translate in a substantially different incidence of postoperative fever. We therefore postulate that duration of surgery is not a major determinant for the occurrence of fever after intraventricular neuroendoscopic procedures. Finally, in our hospital irrigation fluids are always pre-heated to body temperature $\left(37.0-38.0{ }^{\circ} \mathrm{C}\right)$, whereas in the Kinoshita series they were at room temperature (personal communication). We again postulate that irrigation fluid temperature is not a major determinant for the occurrence of fever after intraventricular neuroendoscopic procedures. Finally, all children in the present series had an ETV with or without additional procedures such as cyst puncture or tumor biopsy, whereas in the Kinoshita series 17 patients $(21 \%)$ did not have an ETV. Therefore, at least in our series, we cannot exclude ETV was the sole responsible for the development of fever that may not develop in other intraventricular neuroendoscopic procedures without ETV.

\section{Conclusion}

To the best of our knowledge, we present the first exclusively pediatric patient series on fever following intraventricular neuroendoscopic procedures. Although we believe the quality of the data in this retrospective study is good (after elimination of 9 procedures because of insufficient data), it remains difficult to draw solid conclusions with regard to the factors that may influence fever occurring postoperatively. However, from this study, we learn that fever frequently develops after intraventricular neuroendoscopic procedures in children, especially those above 1 year of age. It follows a rather predictable course, peaking the day of surgery and/or the next day and rapidly subsiding usually within 72 hours. Moreover, these patients are typically not clinically ill. Therefore, fever by itself is not a cardinal symptom the first few days postoperatively, except when combined with other symptoms and signs in children who are clearly clinically ill. Close observation may suffice for those who are not clinically ill, whereas extra attention should be paid to those whose temperature rises after day 2 especially when clinically ill, as they likely suffer a serious illness. We hope a better understanding of the phenomenon of fever after intraventricular neuroendoscopic procedures will help the attending physicians to avoid unnecessary and especially invasive diagnostic procedures. We recommend to closely observe children after any intraventricular neuroendoscopic procedure for at least 5 days.

\section{Compliance with ethical standards}

Conflict of interest The authors declare they have no conflict of interest.

Open Access This article is distributed under the terms of the Creative Commons Attribution 4.0 International License (http:/ creativecommons.org/licenses/by/4.0/), which permits unrestricted use, distribution, and reproduction in any medium, provided you give appropriate credit to the original author(s) and the source, provide a link to the Creative Commons license, and indicate if changes were made.

\section{References}

1. Furlanetti LL, Santos MV, De Oliveira RS (2013) The success of endoscopic third ventriculostomy in children: analysis of prognostic factors. Pediatr Neurosurg 48:352-359

2. Lam S, Harris D, Rocque B, Ham S (2014) Pediatric endoscopic third ventriculostomy: a population-based study. J Neurosurg Pediatr 14:455-464

3. Husain M, Jha D, DK V, Thaman D, Gupta A, Husain N, et al. (2003) Neuro-endoscopic surgery-experience and outcome analysis of 102 consecutive procedures in a busy neurosurgical centre of India. Acta Neurochir. (Wien) 145:369-375 discussion $375-6$ 
4. Kliegman RM, Behrman RE, Jenson HB, Stanton BF. Nelson Textbook of Pediatrics. 18 edition. Elsevier Health Sciences; 2007.

5. Kinoshita Y, Tominaga A, Saitoh T, Usui S, Takayasu T, Arita K, et al. (2014) Postoperative fever specific to neuroendoscopic procedures. Neurosurg Rev 37:99-104

6. Koch D, Wagner W (2004) Endoscopic third ventriculostomy in infants of less than 1 year of age: which factors influence the outcome? Childs Nerv Syst 20:405-411

7. Zhao P, Wang X, Li C, Gui S, Zong X, Zhang Y (2013) The effectiveness of neuroendoscopic versus non-neuroendoscopic procedures in the treatment of lateral ventricular cysts: a retrospective medical record review study. BMC Neurol 13:59

8. Hailong F, Guangfu H, Haibin T, Hong P, Yong C, Weidong L, et al. (2008) Endoscopic third ventriculostomy in the management of communicating hydrocephalus: a preliminary study. J Neurosurg 109:923-930
9. Sacko O, Boetto S, Lauwers-Cances V, Dupuy M, Roux F-E (2010) Endoscopic third ventriculostomy: outcome analysis in 368 procedures. J Neurosurg Pediatr 5:68-74

10. Chernov M, Kamikawa S, Yamane F, Ishihara S, Kubo O, Hori T (2006) Neurofiberscopic biopsy of tumors of the pineal region and posterior third ventricle: indications, technique, complications, and results. Neurosurgery 59:267-277

11. Simon H (1993) Hyperthermia. N Engl J Med 329:483-487

12. Saper CB, Lowell BB (2014) The hypothalamus. Curr Biol 24: R1111-R1116

13. Hall JE, Guyton AC. Guyton and Hall textbook of medical physiology. 13th Editi. Saunders; 2015.

14. Repin IS, Kratskin IL (1967) Hypothalamic mechanisms of fever. Neurosci Transl 1:336-340

15. Saunders NR, Liddelow SA, Dziegielewska KM (2012) Barrier mechanisms in the developing brain. Front. Pharmacol 3:46 